\title{
Article
}

\section{An Octopus-Inspired Bionic Flexible Gripper for Apple Grasping}

\author{
Jie Pi ${ }^{1}$, Jun Liu ${ }^{1, *}$, Kehong Zhou ${ }^{2}$ and Mingyan Qian ${ }^{1}$ \\ 1 Institute of Agricultural Facilities and Equipment, Jiangsu Academy of Agricultural Sciences, Key Laboratory \\ of Protected Agriculture Engineering in the Middle and Lower Reaches of Yangtze River Ministry of \\ Agriculture and Rural Affairs, Nanjing 210014, China; pijie@jaas.ac.cn (J.P.); 20190977@jaas.ac.cn (M.Q.) \\ 2 Institute of Agricultural Engineering, Jiangsu University, Zhenjiang 212001, China; \\ 2221916050@stmail.ujs.edu.cn \\ * Correspondence: liujun@jaas.ac.cn; Tel.: +025-8439-9439
}

\section{check for}

updates

Citation: Pi, J.; Liu, J.; Zhou, K.; Qian, M. An Octopus-Inspired Bionic Flexible Gripper for Apple Grasping. Agriculture 2021, 11, 1014. https:// doi.org/10.3390/agriculture11101014

Academic Editor: José Pérez-Alonso

Received: 24 August 2021

Accepted: 14 October 2021

Published: 17 October 2021

Publisher's Note: MDPI stays neutral with regard to jurisdictional claims in published maps and institutional affiliations.

Copyright: (c) 2021 by the authors. Licensee MDPI, Basel, Switzerland. This article is an open access article distributed under the terms and conditions of the Creative Commons Attribution (CC BY) license (https:// creativecommons.org/licenses/by/ $4.0 /)$.

\begin{abstract}
When an octopus grasps something, the rigidity of its tentacle can change greatly, which allowing for unlimited freedom, agility, and precision. Inspired by this, a three-finger flexible bionic robot gripper was designed for apple picking. First, a flexible chamber finger was designed to drive the gripper finger to elongate, shorten, and bend, which works through a process of inflating and deflating. Further, we proposed a three-finger mode to achieve two kinds of motion states: grasping and relaxing, by simulating the movement of an octopus grasping at something. In this paper, we evaluated the bending property of the designed flexible bionic gripper through an apple grasping experiment. The experimental results show that the $100.0 \mathrm{~g}$ bionic gripper can load an apple with a weight of $246.5 \sim 350.0 \mathrm{~g}$ and a diameter of $69.0 \sim 99.0 \mathrm{~mm}$, and the grasping success rate is $100 \%$. It has a good grasping performance. Compared to other soft grippers, the proposed bionic flexible gripper has the advantages of being lightweight, and having good cushioning, low driving air pressure, and a strong grasping force.
\end{abstract}

Keywords: octopus tentacles; bionic flexible gripper; pneumatic drive; agriculture

\section{Introduction}

The apple is one of the most common fruits, which possesses huge consumer market, and is available in various kinds, and different weights and sizes. The traditional artificial approaches for picking and sorting imply a shape evaluation process, and the fruit pickers simply sort fruits by judging their shape, weight, and rough skin conditions, thus causing a huge workload in this process. In addition, the cost of apple picking reaches $50 \%$ to $70 \%$ of the total cost [1]. With the development of mechanization and automation technology, apple picking and sorting will gradually rely on mechanical operation, especially in the case of large-scale planting; since apple production is huge, the efficiency and scale of manual picking and sorting simply cannot satisfy the production needs [1]. In order to solve the objective problems, including huge workload, low efficiency, and high cost involved in manual picking and sorting operations, agricultural picking and sorting robots have emerged, becoming one of the research hotspots in the field of artificial intelligence agriculture.

The robot gripper structure is the last part which directly touches the apple, and its excellent performance effectively improves the working efficiency. Since the growing environment of apples is complex, their skin is fragile, and their shape is complex and changeable, it is easy to cause damage to the fruit in the process of picking and sorting, thus affecting the quality, storage, and transportation of apples, and ultimately harming the market price and economic benefits. Traditional industrial robots mainly have a rigid structure, with the characteristics of the "three highs": high stiffness, high strength, and high speed [2]. Thus, it is easy to damage the surface of apples during operation of these 
robots, which requires high precision but results in poor universality. Ma Lvzhong et al. [3] (2009) and Ji Changying et al. [4] (2011) have designed their own apple picking devices, but the structure is complex, which can cause varying degrees of damage to the apple epidermis. Therefore, owing to the particularity and delicacy of apples, soft robots with flexible grippers are more appropriate for apple sorting.

Currently, many scholars and engineers both at home and abroad have started to study the structure of the soft robot. Based on the bionics concept with intelligent materials such as silicone rubber, shape-memory alloys (SMAs) [5], electroactive polymers (EAPs) [6], etc. and new driving technologies such as SMA drivers [7], pneumatic drivers [8,9], magnetorheological drivers [10], EAP drivers, etc., they developed a series of novel robots without adopting the traditional rigid structure, by imitating the structure and behavior of invertebrates in nature, such as earthworms, starfish, octopuses, etc.. This kind of soft robot possesses a strong adaptive ability, and theoretically has infinite freedom, which can be applied to unstructured working environments. In terms of flexible robot manufacturing, many new processing technologies have been introduced, such as shape deposition manufacturing [11,12], 3D printing [13], flexible photolithography technology [14], and composite materials integrated through a variety of manufacturing methods [15]. Currently, led by Harvard University in the United States [13], the research on super-elastic silica gel material as the ontology material, combined with 3D printing technology, is the most popular branch of soft robot research. The GEORGE [16] team at Harvard University (2011) developed a multi-cavity pneumatic software gripper, which has a strong adaptive ability, but it is not good at end bending, thus it is difficult for the gripper to grasp objects. Further, due to the small end contact, instability appears during the working process. The PneuNet (pneumatic mesh) structure proposed by Shepherd et al. [17] from Harvard University is a flexible, multi-cavity pneumatic robot, which is capable of bending. Belgian company Octinion [18] developed a small strawberry picking robot, which is composed of two soft fingers, produced through 3D printing. After grasping the object, the grip will passively deform. Compared with human fingers, it has a larger contact area, and better pressure distribution. Hemming's team [19] designed a sweet pepper-picking claw with a fin-bar structure, which adopts fin-bar fingers and has a fin-bar effect, able bend according to the shape of the object's surface. Dimeas [20] (2015) et al. proposed a fuzzy controller-based hierarchical control gripper, which is combined with a pressure profile sensor, and can adjust the grasping force, and detect the uneven distribution of dislocation or force on the gripper. Yaguchi et al. [21] (2016) designed a tomato-picking robot with a rotating gripper, which can pick tomatoes within $23.0 \mathrm{~s}$, with a success rate of approximately $62.6 \%$. Paul Glick et al. [22] invented a flexible robot gripper with a gecko-like adhesive, which adopts Van der Waals forces to achieve adhesion, and can grasp large curvature objects with high grasping strength and fast execution speed, but this gripper is not suitable for grasping fragile objects. Tomokazu Takahashi et al. [23,24] designed an octopus-inspired vacuum clamper with miniature raised sections, which can grasp rigid objects with flat surfaces, curved surfaces, uneven surfaces, or grooves. Brown et al. [25,26] developed a bag-like gripper containing ground coffee, which is highly compliant and can conform to the shape of an object, thus wrapping the object safely. It is suitable for grasping all kinds of hard, small objects. The "vacuum cleaner" picking machine developed by Abundant Robotics Company [27] (2017) sucks mature apples from fruit trees through a large suction nozzle, but the machine can easily suck excess branches and leaves into the trachea, causing obstruction. Ma Shaochun et al. [28] (2018) developed a device for apple picking based on negative pressure suction, but the proportion of apples without is only $85 \%$. In addition, the relevant apple-picking devices at home and abroad generally have a large body and a complex structure, so it is difficult to realize the non-destructive harvesting of apples. In addition, the cost is high, which is difficult for Chinese fruit farmers to bear.

In conclusion, despite the soft robot becoming a popular research topic in the field of robotics at home and abroad, it is still in the basic research stage in the field of agriculture, 
especially concerning the picking and sorting of fruits and vegetables, and is not widely used. Due to the good qualities of soft robots, developing a flexible gripper with adaptive abilities is the inevitable trend of agricultural intelligent robot development. In China, a bionic soft robots laboratory has been established in the Beijing University of Aeronautics and Astronautics, which has produced several kinds of soft robot for the purpose of grasping [29,30].

The flexible clamping device for apple gathering presented in this paper has the advantages of having a simple structure, convenient fabrication, and low cost. It is verified through experiments that the apple surface is not damaged. Based on an analysis of the muscles of octopus tentacles, we propose a novel bionic flexible agriculture grip structure for apple grasping, according to its structure and motion characteristics. The proposed flexible grip consists of three flexible fingers with the same structures, which are pneumatically controlled. Under certain pressures, the grip can practice two kinds of motion: grasping, and relaxing. In this paper, some assumptions are made on this flexible gripper. Through finite element analysis and experimental analysis, the experimental study of the proposed flexible gripper is conducted, and the conclusion is drawn. It is expected to provide reference for the development of non-destructive apple picking devices, imitating human hands.

\section{Materials and Methods}

Bionics' aim is to imitate the function and behavior of biological systems, that is, to improve technology based on inspiration from nature, and achieve effective applications in engineering. In this paper, a novel bionic flexible gripper was designed based on the principle of bionics, inspired by the structure and movement characteristics of octopus tentacles. The proposed gripper is mainly used for picking and sorting apples.

\subsection{Analysis of the Tentacle Structure of Octopus}

The octopus is an invertebrate marine animal with high agility and intelligence [31], as well as strong ability to learn. Most of the neurons in an octopus (about 60\%) are distributed throughout its eight tentacles, forming an independent nerve center with an infinite degree of freedom, which can provide flexible changes of direction [32], so as to achieve free bending, stretching, winding, and other complex actions.

The movement pattern of octopus tentacles is from the roots to the ends, which is a kind of mobile bending. In any position of the tentacles, a combination of four basic movements may occur: elongation, shortening, twisting, and bending [33]. An octopus catches its prey using the method of enveloping grasp, that is, the tentacles stretch from its roots to contact the object, further cover the object until the tips of the tentacles touch the object, and completely wrap it up. In this way, an octopus can grasp an object without knowing its shape. This complex movement pattern of octopus tentacles is enabled by their unique muscle tissue structure. Its movement pattern and muscle tissue structure support are both based on the muscular hydrostatic skeleton, the interior of which is completely composed of arrays of muscle fibers and connective tissues of collagen fibers arranged in different directions [34]. This dense array of muscle fibers cannot be compressed, and it is difficult to compress its volume with external pressure, i.e., it has volume invariance. Volume invariance is one of the key biological properties of octopus tentacles, enabling them to achieve complex movements. The muscle tissues of octopus tentacles can be divided into radial, longitudinal, and oblique muscles, as shown in Figure 1. When one side of the longitudinal muscle is shortened, the diameter of the tentacle will increase, while the other muscle groups will work together to maintain the diameter of the tentacle. When one side of the longitudinal muscle is shortened by resistance, it causes the other side of the longitudinal muscle to stretch passively, which results in the bending motion along the direction of contraction force, and making the tentacle bend flexibly in the space form. With the cooperation of various muscle tissues, the diameter of octopus tentacles remains 
basically unchanged, and the contraction of the longitudinal muscle on one side can only cause slight irregular bending [35].

The muscular system of an octopus can act as a dynamic skeleton, which allows smooth changes in shape, thus resulting in potentially large movements of the tentacles [36].

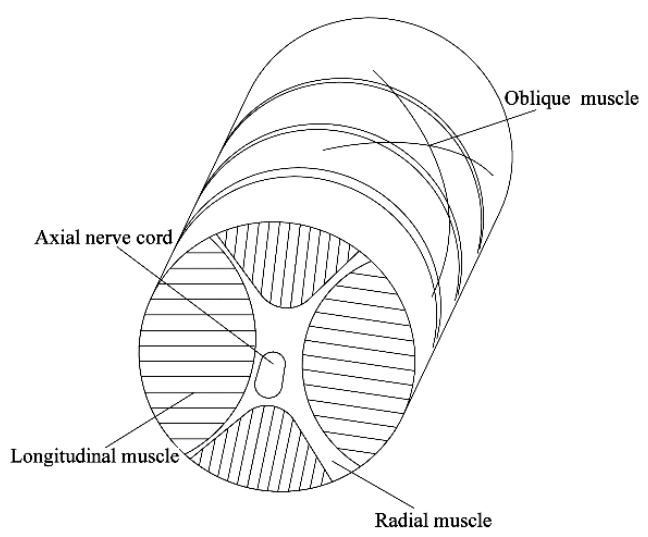

Figure 1. Muscle structure of octopus tentacles.

In order to simulate the muscle characteristics and movement of octopus tentacles, we designed a flexible fluid actuator, which can achieve the two motions of grasping and relaxing via gas driving of the extension, shortening, and bending actions of the grippers.

\subsection{Analysis of Apple Morphology}

The shape of the flexible gripper is designed according to the shape characteristics of apples, based on the principle to minimize the size of gripper. In the process of grasping the apple, the flexible gripper implements wrapped grasping by deformation of the flexible fingers through bending, and the gripper requires a certain deformation space. In order to better fit the shape of grasped target, it is necessary to pre-analyze the shape characteristics of the target, pre-determine the size of the required space, and estimate the deformation volume of the gripper, so as to set the range of clamping deformation and the angle of gripper.

Since the size and shape of apples vary in different stages of growth, the characteristic parameters considered in the design of the clamping claws are of apples in the ripening stage, including geometric parameters, such as mass, transverse diameter, longitudinal diameter, fruit shape index, and minimum enclosing rectangle, as shown in Figure 2.

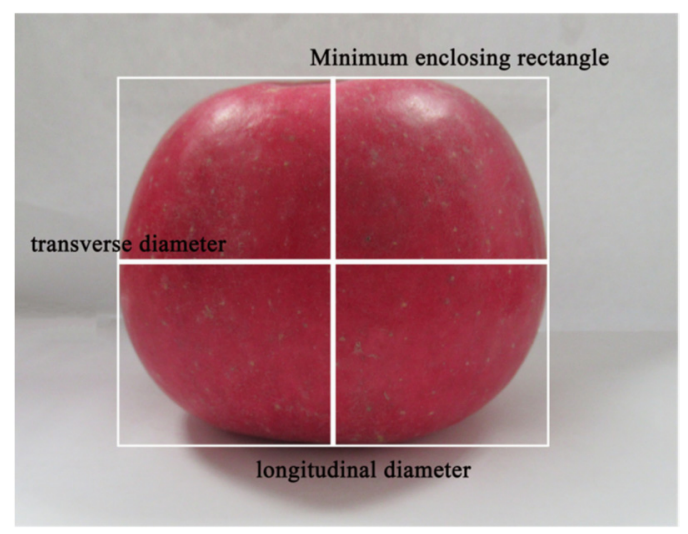

Figure 2. Apple shape measurement.

With the Fuji apple (producing area: Yantai, Shandong Province, China) as a representative sample, according to the shape selection rules, 100 apples with no mechanical damage, plant diseases, or insect pests were selected as the test samples, and were then 
numbered from 1 to 100 . The disk scales (range of $500 \mathrm{~g}$ and precision of $0.1 \mathrm{~g}$ ) and digital caliper (range of $150 \mathrm{~mm}$ and precision of $0.02 \mathrm{~mm}$ ) were adopted for precision measurement and calculation of the sample quality and the shape parameters. Table 1 shows the related characteristic parameters of apple.

According to Table 1, the variation coefficients of apple mass, vertical axis diameter, horizontal axis diameter, and fruit shape indexes are $0.09,0.05,0.04$, and 0.05 , respectively, all of which are small, indicating that the samples have high quality on average, and the dispersion degree is minor.

Table 1. Characteristic parameters of sample apples.

\begin{tabular}{cccccc}
\hline Indicators & $\begin{array}{c}\text { Quality } \\
\mathbf{m} / \mathbf{g}\end{array}$ & $\begin{array}{c}\text { Vertical Axis } \\
\text { Diameter } \\
\mathbf{D}_{\mathbf{v a}} / \mathbf{m m}\end{array}$ & $\begin{array}{c}\text { Horizontal Axis } \\
\text { Diameter } \\
\mathbf{D}_{\mathbf{h a}} / \mathbf{m m}\end{array}$ & $\begin{array}{c}\text { Fruit Shape } \\
\text { Index } \\
\mathbf{D}_{\mathbf{v a}} / \mathbf{D}_{\mathbf{h a}}\end{array}$ & $\begin{array}{c}\text { Minimum Enclosing } \\
\text { Rectangle } \\
\mathbf{A} * \mathbf{b}(\mathbf{m m})\end{array}$ \\
\hline Maximum & 350.0 & 75.0 & 99.0 & 0.76 & $75 * 99$ \\
Minimum & 246.5 & 69.0 & 82.0 & 0.94 & $69 * 82$ \\
Average & 288.2 & 74.5 & 86.9 & 0.86 & - \\
Standard deviation & 24.8 & 3.7 & 3.2 & 0.05 & - \\
Coefficient of variation & 0.09 & 0.05 & 0.04 & 0.05 & - \\
\hline
\end{tabular}

According to the above analysis, the basic indexes of the flexible clamping claw are put forward: the flexible clamping claw adopts the form of three fingers; the initial opening diameter of the clamping claw is $100 \mathrm{~mm}$; the effective deformation length of the finger is more than $90 \mathrm{~mm}$; the load capacity is higher than $0.5 \mathrm{~kg}$; and, within the range of operating temperatures $\left(-25 \sim 60^{\circ} \mathrm{C}\right)$, it presents good adaptive deformation and flexible bending deformation capacity.

\subsection{Design and Manufacture of a Single Flexible Finger}

In order to simulate the particular texture and structure of octopus tentacles, this paper adopts the silicone rubber Dragonskin 30-a viscoelastic material一to create the flexible fingers, and a pneumatic driver is employed in the simulation.

A single flexible finger is composed of a top layer with a balloon structure, and a bottom layer with poor deformation capacity, as shown in Figure 3a. The top layer has the structure of a hollow elastic chamber, connected to the vent hole at the tip of finger, which is then connected to the external air source through an air pipe. A single flexible finger has 7 chambers in total, each of which is $7 \mathrm{~mm}$ wide and $13 \mathrm{~mm}$ high, with a wall thickness of $1.5 \mathrm{~mm}$. Without deformation, the finger is $104 \mathrm{~mm}$ long, and the underside of the finger is $4 \mathrm{~mm}$ thick. A fixed structure is situated at the root of the finger, as shown in Figure 3b. In the initial state of the finger, the chamber pressure is consistent with the external atmospheric pressure, and the finger remains upright. As the finger is driven pneumatically, it is filled with gas, the pressure in the chamber becomes greater than the external pressure, and the chamber on the top layer of the finger expands, thus elongating the top part of the finger. However, due to the thicker bottom layer, the deformation capacity of the finger is limited. After removal of the gas, the pressures in and out of the chamber are once again equal, and the finger is restored to its original shape. Similarly, if the finger is pumped and decompressed, the chamber contracts, both layers of the finger are shortened, forcing the finger to bend outward. The deformation process of the chamber is shown in Figure 4. 


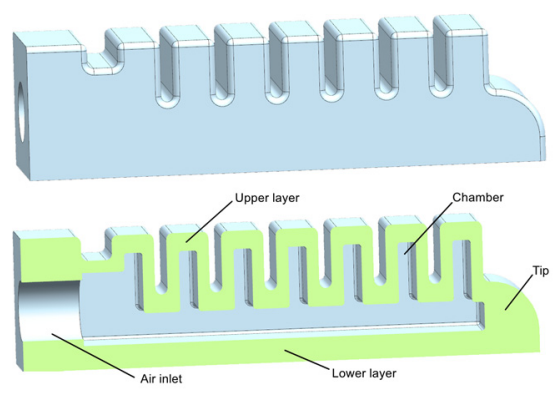

(a)

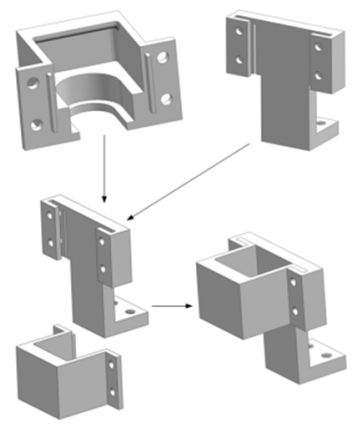

(b)

Figure 3. Schematic diagram of flexible finger: (a) schematic diagram of finger; (b) schematic diagram of fixed structure.
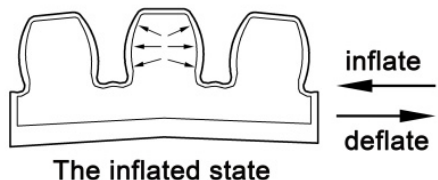

deflate

The inflated state

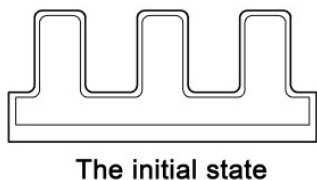

The initial state

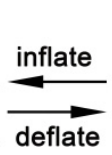

deflate

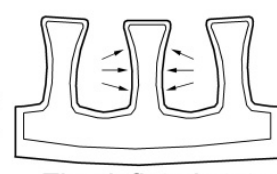

The deflated state

Figure 4. Schematic diagram of chamber deformation process.

The flexible pneumatic finger can be produced through 3D printing, which is more efficient and faster than other production methods. It has good integrity, and includes features of high strength and high tolerance to expansion pressure, meaning that the gripper can obtain greater grasping force. Therefore, the flexible gripper designed in this paper was also created through 3D printing technology.

\subsection{Finite-Element Analysis and Experimental Analysis of Flexible Finger}

The finite-element analysis software ANSYS (ANSYSV17.0) was used for the finiteelement simulation and analysis. The material parameters for each part of the flexible finger are shown in Table 2. Firstly, the model of a single flexible finger was established by using the UG (Unigraphics NX) software, as shown in Figure 5a, and then the model was imported into ANSYS for simulation. The static structural module was created in ANSYS Workbench; subsequently, the material properties were integrated to obtain the ANSYS model, as shown in Figure 5b. By refining the grid and simplifying the model, the precision of the grid was determined, and 160,935 nodes and 62,146 elements were divided, as shown in Figure $5 \mathrm{c}$. The load of $65 \mathrm{kPa}$ and $-65 \mathrm{kPa}$ were added to each surface of the flexible finger, the fixed structure was set as rigid material, and the deformation was neglected. After fixing the end of the finger, the deformation data was obtained, based on the displacement of flexible finger. The simulation results are shown in Figure 6. The simulation results show that: under the pressure of $65 \mathrm{kpa}$, the bending angle of the flexible finger was $27.19^{\circ}(\alpha)$ and the displacement of the fingertip was $31.871 \mathrm{~mm}(\Delta \mathrm{s} 1)$. Under the pressure of $-65 \mathrm{kpa}$, the bending angle of the flexible finger was $16.82^{\circ}(\beta)$, and the displacement of the fingertip was $21.679 \mathrm{~mm}(\Delta \mathrm{s} 2)$.

Table 2. Material parameters.

\begin{tabular}{cccccc}
\hline Name & Material Type & Density $/\left(\mathbf{k g} \cdot \mathbf{m}^{-3}\right)$ & \multicolumn{3}{c}{ Other Parameters } \\
\hline Flexible fingers & Flexible & $1.08 \times 10^{9}$ & $\mu_{1}=0.264 \mathrm{MPa}$ & $\alpha_{1}=3.0158$ & $\mathrm{D}_{1}=0$ \\
\hline Fixed structure & Fixed & 7850 & Modulus of elasticity: & $2 \times 10^{5} \mathrm{MPa}$ & $\begin{array}{c}\text { Poisson's ratio: } \\
0.3\end{array}$ \\
\hline
\end{tabular}


(a)

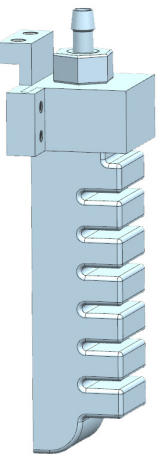

(b)

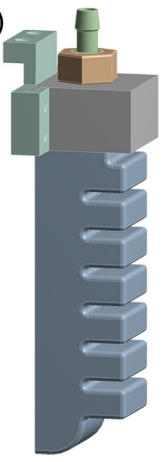

(c)

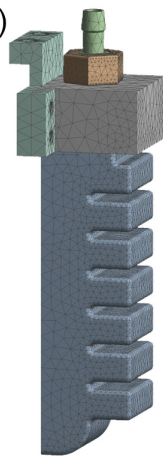

Figure 5. Model flexible finger: (a) UG model; (b) ANSYS model; (c) grid chart.

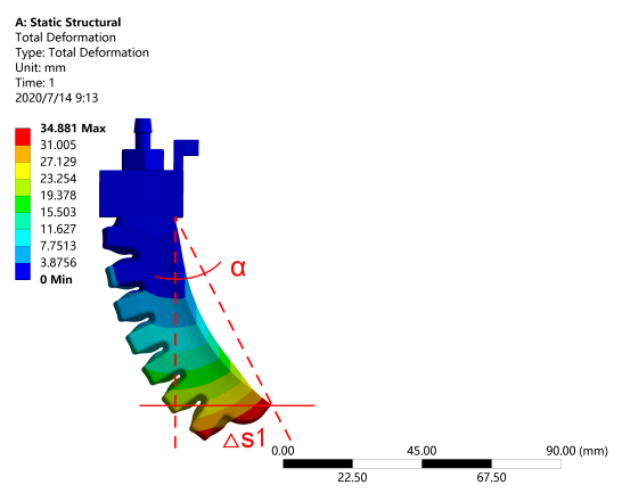

(a)

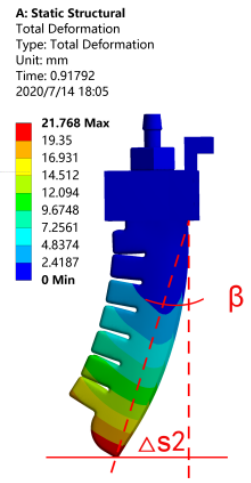

$20.000 .000 .000 .000(\mathrm{~mm})$

(b)

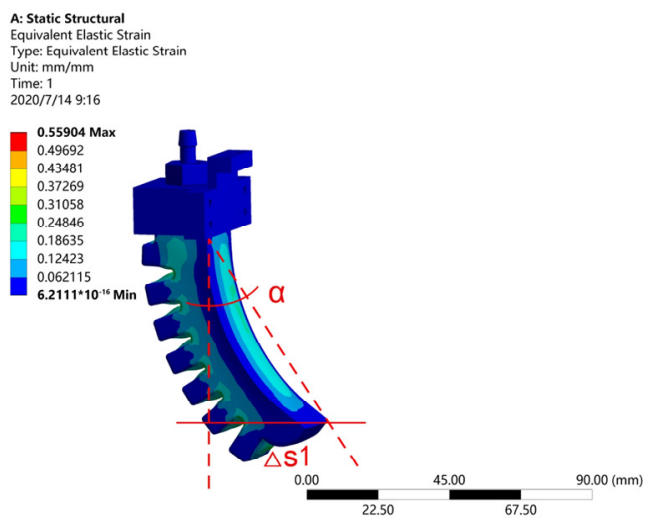

(c)

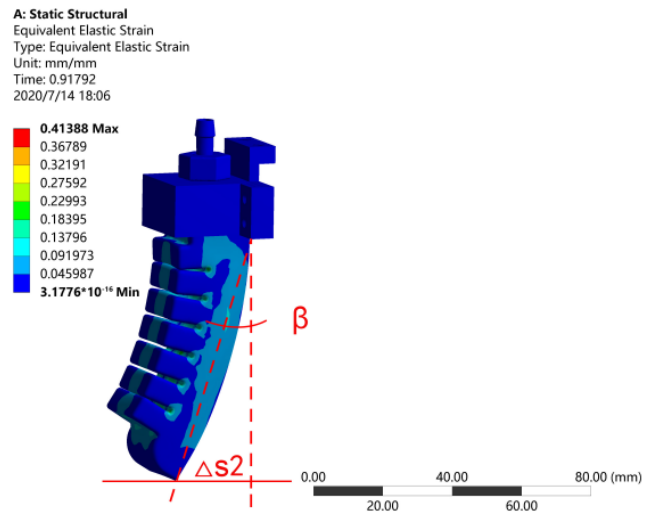

(d)

Figure 6. Simulation results. $(\mathbf{a}, \mathbf{c})$ show the deformation diagram and the stress diagram after inflation; (b,d) show the deformation diagram and the stress diagram after deflation.

The air path of the flexible finger was connected to the circuit to conduct the inflation and deflation experiments. The results are shown in Figure 7 . The results show that: under the pressure of $65 \mathrm{kpa}$, the bending angle of the flexible finger was $28.05^{\circ}(\alpha)$, and the displacement of the fingertip was $32.948 \mathrm{~mm}(\Delta \mathrm{s} 1)$. Under the pressure of $-65 \mathrm{kpa}$, the bending angle of the flexible finger was $17.35^{\circ}(\beta)$, and the displacement of the fingertip was $23.029 \mathrm{~mm}(\Delta \mathrm{s} 2)$. 


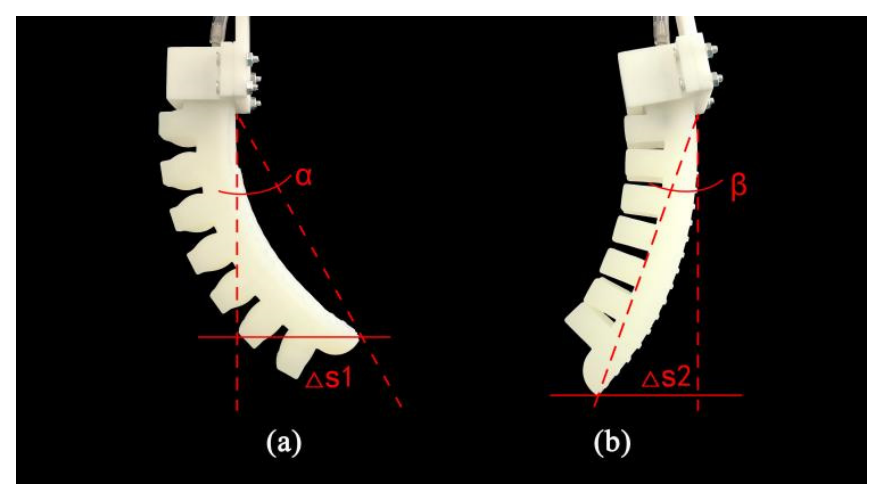

Figure 7. Experimental results: (a) inflation status; (b) deflation state.

It can be seen from Figures 6 and 7 that the simulation results are highly consistent with the experimental results, which proves the feasibility of the flexible finger, and the accuracy of the simulation model.

Since the bending capacity of flexible fingers has a greater influence on the structural design, in order to give the flexible gripper better bending deformation and fetching performance, the optimization structural design for flexible fingers is conducted based on the simulation model in Section 2.3. The main parameters of the original finger model are as follows: chamber spacing $2 \mathrm{~mm}$; 7 chambers; chamber thickness $7 \mathrm{~mm}$; chamber wall thickness $1.5 \mathrm{~mm}$; finger side thickness $4 \mathrm{~mm}$; finger underside thickness $4 \mathrm{~mm}$. The control variable method was employed to change the factors which impact the length of flexible finger (spacing of chambers, number of chambers, thickness of chambers), the wall thickness of chambers, the thickness of lower layer, and other factors, and to compare the horizontal displacement at the end of the fingers. The larger the horizontal displacement, the greater the bending degree of the finger. The finger variable factors are shown in Figure 8.

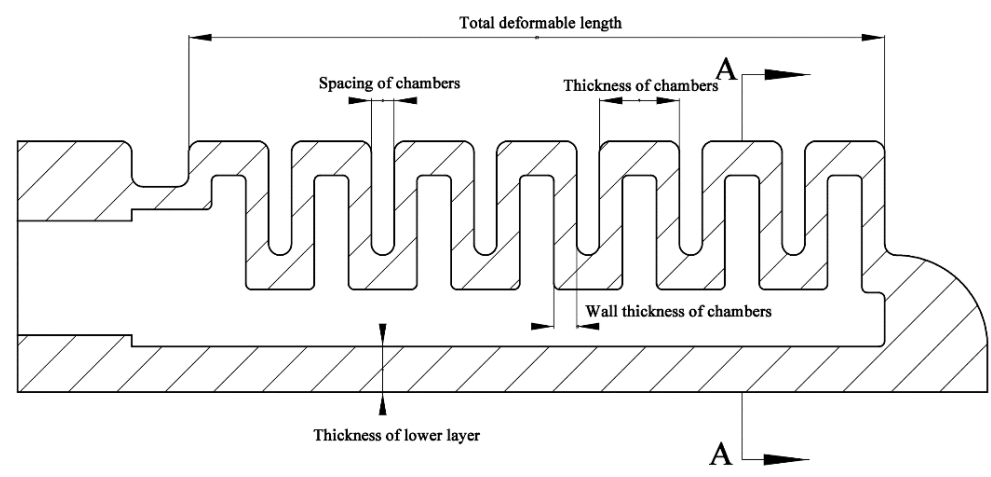

A-A

Figure 8. Finger variable factors.

\subsection{Experiment of Flexible Gripper}

The bionic flexible gripper designed in this work is a three-finger gripper, in order to present contact forces evenly. A flange structure is incorporated to fix the three flexible fingers. The quality of the flexible gripper is $100 \mathrm{~g}$, excluding the flange structure. The structure diagram of the flexible gripper is shown in Figure 9. Both the flange structure and the flexible fingers are produced through 3D printing. 


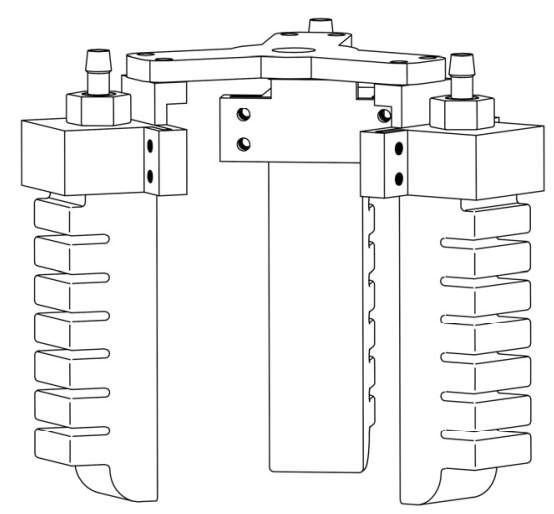

Figure 9. Structure diagram of flexible gripper.

As shown in Figure 10, the upper limit of the object mass that can be grasped by the gripper in different air pressure ranges is tested by adding a weight in the circular sphere.

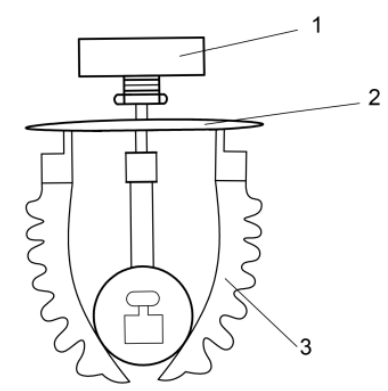

Figure 10. Maximum gripping quality test device of flexible gripper. 1: manipulator; 2: flange plate; 3: flexible finger.

\subsubsection{Experiment of Apple Grabbing}

In order to test the grasping ability of the gripper, an apple grasping experiment was carried out in a laboratory environment to evaluate it. The flexible gripper was fixed on the six-axis manipulator. The finger movement was accomplished with a pneumatic actuator. We connected the gas circuit and electric circuit to carry out the grasping experiments; the grasped object is the apple mentioned above.

We determined the initial position point $\mathrm{A}$ and the terminal position point $\mathrm{B}$ on the experimental platform, as shown in Figure 11a. The flexible gripper opened the finger to grab the apple from point $A$, and then the manipulator moved up $100 \mathrm{~mm}$. The manipulator rotated horizontally above point $B$, and the manipulator moved down to point $B$ to relax the flexible gripper, successfully completing the experiment. The motion path of the manipulator is shown in Figure 11b.

Apples numbered from 1 to 90 were selected for grab test, and apples numbered from 91 to 100 were taken as untreated comparison items, and kept in their original state. The working pressure was set to $50 \mathrm{kpa}$, and the working speed of the manipulator was set to $480 \mathrm{~mm} / \mathrm{s}$. The continuous loading time from the touch of the finger with the apple to the stable grasping of the apple was $3 \mathrm{~s}$. Upon completion of each grab, the contact area was marked on the apple. The number of successful experiments and the number of failures were recorded, and the grasping success rate was calculated. 


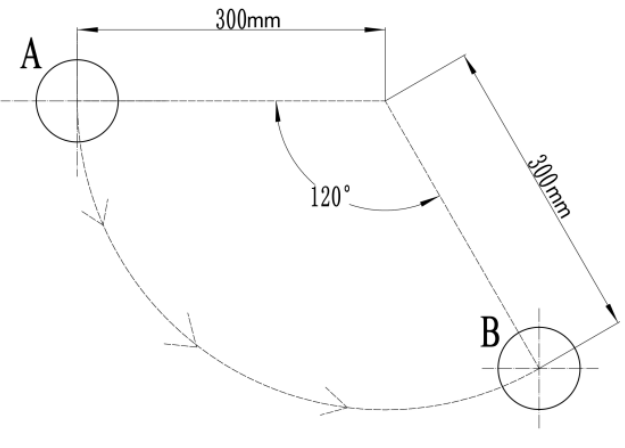

(a)

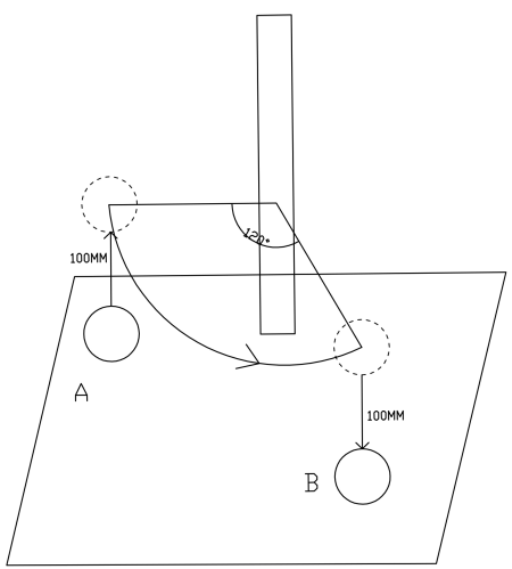

(b)

Figure 11. Motion path of the manipulator. (a) schematic diagram of rotation angle of manipulator; (b) schematic diagram of manipulator motion path.

\subsubsection{Experiment of Apple Damage}

In order to observe the damage induced on apples grabbed by the flexible gripper, we carried out an apple damage analysis.

(1) After completing the apple grab experiment, a $5 \times$ magnifying glass was used to observe whether there was any skin damage or juice overflow in the contact area between the apple and finger;

(2) The apple numbered 91 was selected, and grasped with a rigid claw. Subsequently, 10 apples were randomly selected from the successful grasping experiment. The surfaces which were touched by the finger were sliced respectively. The thickness was $2 \mathrm{~mm}$, and placed under $5 \times$ microscope.

(3) 20 apples were randomly selected from the successful grasping experiment, and 9 untested apples numbered $92-100$ were stored in the same environment. The temperature was maintained at $25^{\circ} \mathrm{C}$, and the humidity at $80 \%$. After seven days, the surface condition of the apples was observed. The contact area between the apple and finger was peeled to observe the internal pulp.

\section{Results and Discussion}

\subsection{Simulation Results}

As shown in Table 3 and Figure 12a, three factors (spacing of chambers, number of chambers, and thickness of chambers) that affect the length of the finger were compared, and the main parameters of the original finger model were kept as follows: chamber wall thickness $1.5 \mathrm{~mm}$; finger side thickness $4 \mathrm{~mm}$; and finger lower thickness $4 \mathrm{~mm}$. With the same amount of increase in the length of the finger, the horizontal displacement of the finger end presented a similar change, and the finger bending also changed with the same degree. Therefore, it is inferred that, when the wall thickness of finger is constant, the increase in finger length will lead to the increase in the degree of finger bending, and the degree of change is not significantly affected by the change of finger length.

We changed the thickness of the lower layer, and kept the main parameters of the original finger model as follows: chamber spacing $2 \mathrm{~mm}$; 7 chambers; chamber thickness $7 \mathrm{~mm}$; chamber wall thickness $1.5 \mathrm{~mm}$; and finger lower thickness $4 \mathrm{~mm}$. As shown in Table 4 and Figure 12b, the horizontal displacement of the finger end and the thickness of the lower layer present approximately linear change. The horizontal displacement decreases by approximately $2 \mathrm{~mm}$ for every $1 \mathrm{~mm}$ of increase in the thickness of lower layer. As the thickness of the lower layer increases, the horizontal displacement of the finger end also declines, and such decrease gradually becomes gentler. When the lower 
layer is $2 \mathrm{~mm}$ thick, the bending deformation of the finger is significant, and the underside of the finger shows transverse deformation, protruding outwards.

Table 3. Analysis of simulation results (spacing of chambers, number of chambers, thickness of chambers). The bold part indicates the variable factors in the experiment.

\begin{tabular}{ccccc}
\hline $\begin{array}{c}\text { Number of } \\
\text { Chambers/Units }\end{array}$ & $\begin{array}{c}\text { Spacing of } \\
\text { Chambers/mm }\end{array}$ & $\begin{array}{c}\text { Thickness of } \\
\text { Chambers/ } \\
\text { mm }\end{array}$ & $\begin{array}{c}\text { Total Length/ } \\
\text { mm }\end{array}$ & $\begin{array}{c}\text { Horizontal } \\
\text { Displacement } \\
\text { of Finger End/ } \\
\text { mm }\end{array}$ \\
\hline $\mathbf{7}$ & 2 & 7 & 76 & 15.791 \\
$\mathbf{8}$ & 2 & 7 & 85 & 20.434 \\
$\mathbf{9}$ & 2 & 7 & 94 & 25.719 \\
$\mathbf{1 0}$ & 2 & 7 & 103 & 31.468 \\
$\mathbf{1 1}$ & 2 & 7 & 112 & 37.841 \\
\hline 7 & $\mathbf{1}$ & 7 & 86 & 20.824 \\
7 & $\mathbf{2}$ & 7 & 94 & 25.719 \\
7 & $\mathbf{3}$ & 7 & 102 & 30.286 \\
7 & $\mathbf{4}$ & 7 & 110 & 35.053 \\
7 & $\mathbf{5}$ & $\mathbf{6}$ & 118 & 40.131 \\
\hline 7 & 2 & $\mathbf{7}$ & 85 & 20.824 \\
7 & 2 & $\mathbf{8}$ & 94 & 25.719 \\
7 & 2 & $\mathbf{9}$ & 112 & 30.286 \\
7 & 2 & $\mathbf{1 0}$ & 121 & 35.053 \\
7 & 2 & &
\end{tabular}

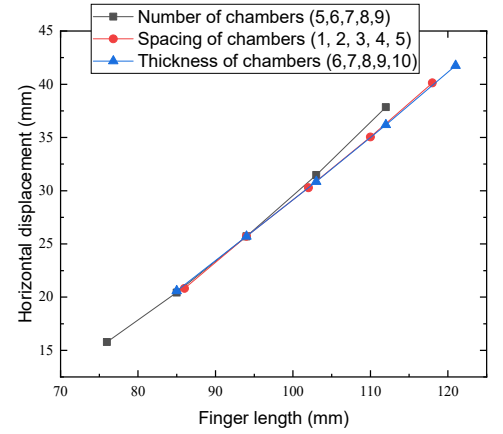

(a)

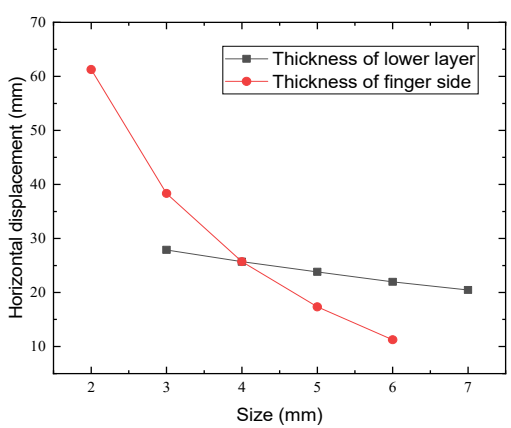

(b)

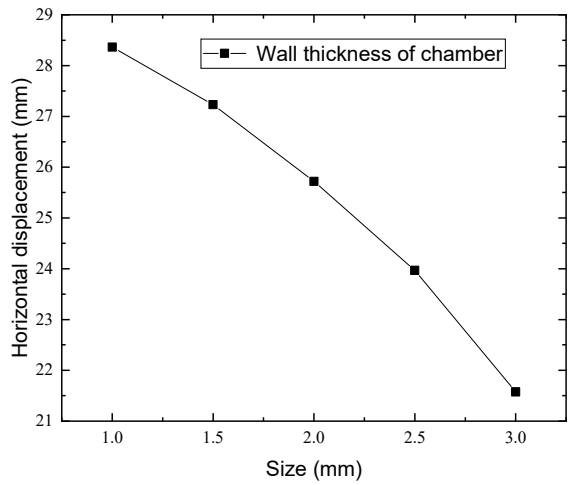

(c)

Figure 12. Simulation results: (a) shows the relationship between the spacing of chambers, the number of chambers, the thickness of chambers, and the horizontal displacement of finger ends; (b) shows the relationship between the thickness of finger bottom and the horizontal displacement of finger ends; (c) shows the relationship between the wall thickness of chambers and the horizontal displacement of finger ends. 
Table 4. Analysis of simulation results (thickness of lower layer, the thickness of finger side, wall thickness of chambers). The bold part indicates the variable factors in the experiment.

\begin{tabular}{cccc}
\hline $\begin{array}{c}\text { Thickness of Lower } \\
\text { Layer/ } \\
\text { mm }\end{array}$ & $\begin{array}{c}\text { Thickness of Finger } \\
\text { Side/ } \\
\text { mm }\end{array}$ & $\begin{array}{c}\text { Wall Thickness of } \\
\text { Chambers/ } \\
\text { mm }\end{array}$ & $\begin{array}{c}\text { Horizontal } \\
\text { Displacement of } \\
\text { Finger End/ } \\
\text { mm }\end{array}$ \\
\hline $\mathbf{3}$ & 4 & 1.5 & 27.882 \\
$\mathbf{4}$ & 4 & 1.5 & 25.719 \\
$\mathbf{5}$ & 4 & 1.5 & 23.814 \\
$\mathbf{6}$ & 4 & 1.5 & 21.977 \\
$\mathbf{7}$ & 4 & 1.5 & 20.467 \\
\hline 4 & $\mathbf{2}$ & 1.5 & 20.824 \\
4 & $\mathbf{3}$ & 1.5 & 25.719 \\
4 & $\mathbf{4}$ & 1.5 & 30.286 \\
4 & $\mathbf{5}$ & 1.5 & 35.053 \\
4 & $\mathbf{6}$ & 1.5 & 40.131 \\
\hline 4 & 4 & $\mathbf{1 . 0}$ & 20.824 \\
4 & 4 & $\mathbf{1 . 5}$ & 25.719 \\
4 & 4 & $\mathbf{2 . 0}$ & 30.286 \\
4 & 4 & $\mathbf{2 . 5}$ & 35.053 \\
4 & 4 & $\mathbf{3 . 0}$ & 40.131 \\
\hline
\end{tabular}

We changed the thickness of finger side, and kept the main parameters of the original finger model as follows: chamber spacing $2 \mathrm{~mm} ; 7$ chambers; chamber thickness $7 \mathrm{~mm}$; chamber wall thickness $1.5 \mathrm{~mm}$; and finger side thickness $4 \mathrm{~mm}$. As shown in Table 4 and Figure 12b, the horizontal displacement of the finger end and the thickness of the finger underside present approximately linear change. The horizontal displacement decreases by approximately $2 \mathrm{~mm}$ for every $1 \mathrm{~mm}$ of increase in the thickness of finger underside. As the thickness of the finger underside decreases, the horizontal displacement of the finger end also declines, and such decrease gradually becomes gentler. When the finger underside is $2 \mathrm{~mm}$ thick, the bending deformation of the finger is significant, and the underside of the finger shows transverse deformation, protruding outwards.

We changed the wall thickness of the chambers and the main parameters of the original finger model as follows: chamber spacing $2 \mathrm{~mm}$; 7 chambers; chamber thickness $7 \mathrm{~mm}$; finger side thickness $4 \mathrm{~mm}$; and finger lower thickness $4 \mathrm{~mm}$. As shown Table 4 and in Figure 12c, with the increase of the wall thickness of the flexible finger chambers, the horizontal displacement gradually decreases at increasingly higher rate. This is because when the balloon wall thickness increases, the thin wall can bear the same pressure, and the volume of deformation will decrease. The deformation of this kind of pneumatic chamber finger is mainly caused by the deformation of the thin wall of the balloon. Therefore, the deformation degree of the flexible finger will also decrease.

This section may be divided by subheadings. It should provide a concise and precise description of the experimental results and their interpretation, as well as the experimental conclusions that can be drawn.

According to the simulation results above, by comparing the influencing factors on the bending degree of flexible fingers, and based on the minimum size principle for the clip parts of the flexible clamp claw, the structure of the flexible fingers is finally determined. In other words, the structure is a single flexible finger with 7 chambers in total, each measuring $10 \mathrm{~mm}$ wide and $15 \mathrm{~mm}$ high with a wall thickness of $2 \mathrm{~mm}$, with chamber spacing of $4 \mathrm{~mm}$; the thickness of lower layer is $4 \mathrm{~mm}$, the total length of the finger is $116 \mathrm{~mm}$ with no deformation, and the thickness of finger bottom is $4 \mathrm{~mm}$. In order to increase friction, the mesh stripe structure of finger was designed.

\subsection{Experiment Results and Analysis of Flexible Gripper}

The experimental results are shown in Figure 13. The pressure in the finger cavity of 
the flexible gripper is directly proportional to the mass of the grasping target. The maximum mass of the apple used in this paper is $350.0 \mathrm{~g}$, thus the driving air pressure is $50.0 \mathrm{kPa}$, which can meet the requirements.

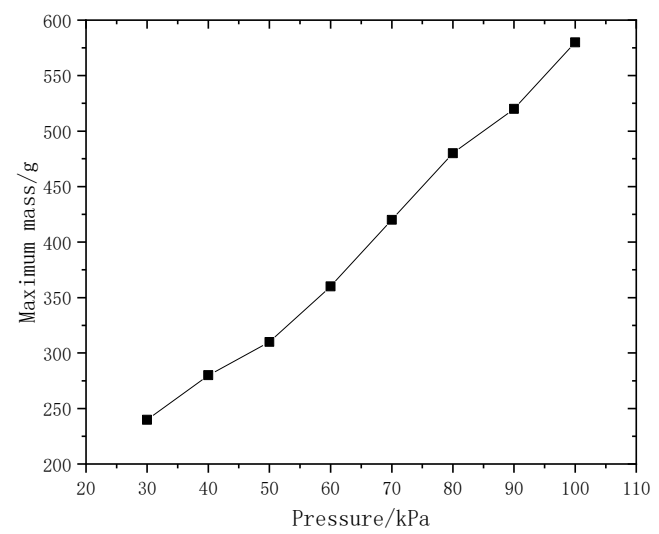

Figure 13. Maximum mass of the flexible gripper can grasp under different air pressures.

The gripper successfully completed the grasping experiment 90 times, failed 0 times, thus the success rate was $100 \%$. It has been proven that the gripper can meet the grasping requirements of conventional apples, and that the grasping effect is good.

\subsection{Experimental Results and Analysis for Apple Damage}

Using a $5 \times$ magnifying glass to observe the apple grabbed by the flexible clamping claw, it can be found that there is no damage to the epidermis at the contact point between the finger and the apple, thus the damage rate is $0 \%$.

The microscopic observation experiment shows that the apple surface cells grasped by the flexible gripper are round and full, and the microstructure is not damaged, as shown in Figure 14a. The apple surface grabbed by the rigid clamping claw produces dark marks, cells are damaged, and the microstructure is damaged, as shown in Figure 14b.
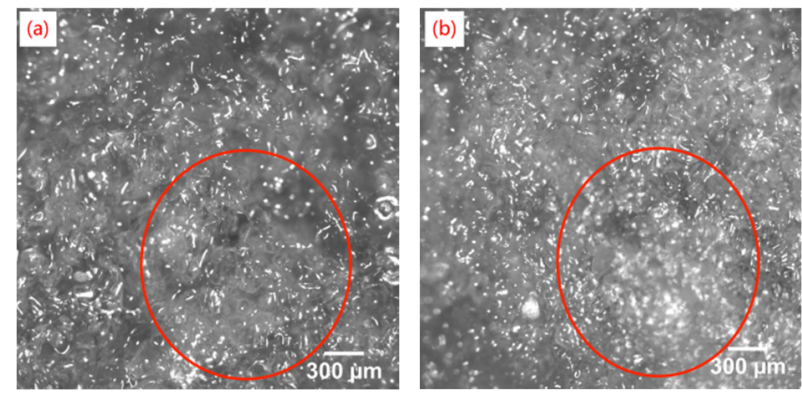

Figure 14. Apple surface under 5 times microscope. (a) apple held by flexible gripper. (b) apple held by rigid gripper.

The apples which had been kept under constant temperature and humidity conditions were removed after seven days. Upon observation of the surface of apple, there was no visible damage on the skin on the contact point between finger and apple, as shown in Figure 15a. After peeling, the internal pulp was observed, and there was no visible, as shown in Figure 15b. It is thus proven that the flexible picking claw does not mechanically damage the apple. 


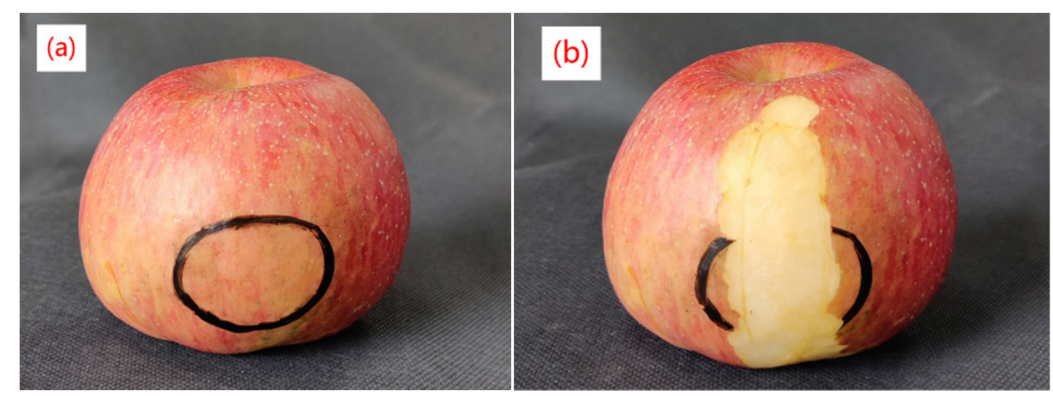

Figure 15. Apples stored for seven days. (a) apple skin condition; (b) apple pulp condition.

At present, there are many soft grippers that can be used for robot harvesting. For different capture targets, each study puts forward different strategies [37]. In many cases, it is difficult to make a comparative evaluation. The current harvesting robots in China, such as the apple-picking robot of Jiangsu University, the elevated strawberry-picking robot of China Agricultural University and the cucumber-picking robot in greenhouses [38], are still in their infancy. At present, SDM, software lithography, and wax loss manufacturing are the most commonly used processing technologies for software robots [11,39]. However, there are many processing procedures, such as long cycle and single material, so it is difficult to process a more complex three-dimensional cavity soft robot. Compared with their manufacturing process, we successfully used silica gel material and the FDM-based $3 \mathrm{D}$ printing technique to fabricate directly bionic flexible grippers with complex inner geometry. In addition, this effectively shortens the production cycle, and reduces cost. Moreover, we have proven through experiments that the proposed bionic flexible gripper has the advantages of being lightweight, and having good cushioning, low driving air pressure, and a strong grasping force.

\section{Conclusions}

This paper presents a bionic flexible gripper inspired by octopus tentacles, used to grasp apples, which is driven pneumatically. Firstly, the muscle structure and movement characteristics of octopus tentacles and the apple shape were analyzed theoretically, and the basic design indexes of flexible finger were put forward. Subsequently, by using the ANSYS finite-element analysis software, a single flexible finger model was established for simulation and optimization of structural design, and the structure of a single flexible finger was determined. Finally, based on the structure of a single flexible finger, the structure of a three-finger flexible gripper was proposed. The gripper is driven by pneumatic force, and the grasping and relaxing motions are realized based on the process of inflation and deflation, so as to complete the grasping and relaxing of the apple.

In order to test the performance of flexible gripper, we conducted a test using the apple gripper. We set the moving path of the flexible gripper after grasping the apple by using the six-axis manipulator, and completed 90 grasping experiments. The apple did not fall during the grasping process, and the success rate was $100 \%$. We conclude that the flexible gripper can successfully grasp apples with a diameter of $69.0 \sim 99.0 \mathrm{~mm}$, and a mass of $246.5 \sim 350.0 \mathrm{~g}$ when the driving air pressure is $50.0 \mathrm{kPa}$. The experiment was completed with great performance.

In order to observe the damage caused to apples grabbed by the flexible gripper, we carried out an apple damage analysis. The experimental results show that the bionic flexible gripper can not only effectively grasp apples, but also cause no damage the apple skin and inner pulp tissues.

The flexible gripper is driven pneumatically, and both the flexible fingers and fixed structure were all produced with 3D printing technology, which has the advantages of strong deformation capacity, durability, convenient manufacturing, and low cost. It is not only suitable for grasping apples, but also for grasping other fruits and vegetables. 
However, our current research work has some limitations. On the one hand, the structural design of the flexible gripper needs further improvement. Since the material, size, and shape of the flexible gripper are important factors that need to be considered during the design of gripper, we will try to make it more universal in our future work, and achieve faster and more stable working conditions. On the other hand, this study only carries out the structural design and experimental verification of the flexible gripper for these two operations, and proves that the bionic gripper we designed can successfully complete the two operations of grasping and loosening an apple without damaging the skin of apple. This is only the first step of our research work. As a future work, we will study the whole flexible picking and sorting robot, including the vision system, positioning system, obstacle avoidance navigation system, and so on.

Author Contributions: Conceptualization, J.P. and J.L.; data curation, K.Z. and M.Q.; writingoriginal draft preparation, J.P.; All authors have read and agreed to the published version of the manuscript.

Funding: This work was funded by Jiangsu Agriculture Science and Technology Innovation Fund [Grant No.CX(21)1007], and the Key Laboratory for Protected Agricultural Engineering in the Middle and Lower Reaches of Yangtze River, Ministry of Agriculture and Rural Affairs.

Institutional Review Board Statement: Not applicable.

Informed Consent Statement: Not applicable.

Data Availability Statement: The data presented in this study are available on request from the corresponding author.

Conflicts of Interest: The authors declare no conflict of interest.

\section{References}

1. Li, J.J. Research on Design Methods of Flexible Grasping Devices with Variable Stiffness; University of Chinese Academy of Sciences: Beijing, China, 2019.

2. Deng, X.L.; Luo, Z.W.; Pang, J.Q.; Zhang, Y.M.; Yang, C.J.; Li, R.Q. Design and experiment of bionic nondestructive handheld suction apple picker. J. China Agric. Univ. 2019, 24, 106-114.

3. Ma, L.Z.; Yang, W.L.; Wang, C.J.; Chen, X.X.; Xue, C.; Lin, Y.Z.; Liu, A.R. Structure design and experiment of the end-effector for apple-harvesting robot. J. Agric. Mech. Res. 2009, 31, 65-67.

4. Zhang, Q.L.; Ji, C.Y.; Gao, F.; Zhao, W.Y. Effect of apple picking manipulator on fruits damage. Sci. Technol. Food Ind. 2011, 32, 404-405.

5. Laschi, C.; Cianchetti, M.; Mazzolai, B.; Margheri, L.; Follador, M.; Dario, P. Soft Robot Arm Inspired by the Octopus. Adv. Robot. 2012, 26, 709-727. [CrossRef]

6. Shen, Q.; Trabia, S.; Stalbaum, T.; Palmre, V.; Kim, K.; Oh, I. A multiple-shape memory polymer-metal composite actuator capable of programmable control, creating complex 3D motion of bending, twisting, and oscillation. Sci. Rep. 2016, 6, 24462. [CrossRef] [PubMed]

7. Cianchetti, M.; Calisti, M.; Margheri, L.; Kuba, M.; Laschi, C. Bioinspired locomotion and grasping in water: The soft eight-arm OCTOPUS robot. Bioinspir. Biomim. 2015, 10, 035003. [CrossRef]

8. Paek, J.; Cho, I.; Kim, J. Microrobotic tentacles with spiral bending capability based on shape-engineered elastomeric microtubes. Sci. Rep. 2015, 5, 10768. [CrossRef]

9. Sun, Y.; Yap, H.K.; Liang, X.; Guo, J.; Qi, P.; Ang, M.H.; Yeow, C.H. Stiffness customization and ratterning for property Modulation of Silicone-Based Soft Pneumatic Actuators. Soft Robot. 2017, 4, 251-260. [CrossRef]

10. Kashima, S.; Miyasaka, F.; Hirata, K. Novel soft actuator using magnetorheological elastomer. IEEE Trans. Magn. 2012, 48, 1649-1652. [CrossRef]

11. Gafford, J.; Ding, Y.; Harris, A.; McKenna, T.; Polygerinos, P.; Holland, D.; Walsh, C.; Moser, A. Shape Deposition Manufacturing of a Soft, Atraumatic, and Deployable Surgical Grasper. J. Mech. Robot. 2015, 7, 021006. [CrossRef]

12. Cho, K.-J.; Koh, J.-S.; Kim, S.; Chu, W.-S.; Hong, Y.; Ahn, S.-H. Review of manufacturing processes for soft biomimetic robots. Int. J. Precis. Eng. Manuf. 2009, 10, 171-181. [CrossRef]

13. Bartlett, N.W.; Tolley, M.T.; Overvelde, J.T.B.; Weaver, J.C.; Mosadegh, B.; Bertoldi, K.; Whitesides, G.M.; Wood, R.J. A 3D-printed, functionally graded soft robot powered by combustion. Science 2015, 349, 161-165. [CrossRef]

14. Morin, S.A.; Shepherd, R.F.; Kwok, S.W.; Stokes, A.A.; Nemiroski, A.; Whitesides, G.M. Camouflage and Display for Soft Machines. Science 2012, 337, 828-832. [CrossRef] 
15. Wehner, M.; Truby, R.L.; Fitzgerald, D.J.; Mosadegh, B.; Whitesides, G.M.; Lewis, J.A.; Wood, R.J. Anintegrated design and fabrication strategy for entirelysoft autonomous robots. Nature 2016, 536, 451-455. [CrossRef]

16. Ilievski, F.; Mazzeo, A.D.; Shepherd, R.; Chen, X.; Whitesides, G.M. Soft Robotics for Chemists. Angew. Chem. Int. Ed. 2011, 50, 1890-1895. [CrossRef] [PubMed]

17. Shepherda, R.F.; Ilievskia, F.; Choia, W.; Morina, S.A.; Stokesa, A.A.; Mazzeoa, A.D.; Chen, X.; Wanga, M.; Whitesides, G.M. Multigait soft robot. Proc. Natl. Acad. Sci. USA 2011, 108, 20400-20403. [CrossRef] [PubMed]

18. Preter, A.D.; Anthonis, J.; Baerdemaeker, J.D. Development of a robot for harvesting strawberries. ScienceDirect 2018, 51, 14-19. [CrossRef]

19. Hemming, J.; Van Tuijl, B.; Gauchel, W.; Wais, E. Field test of different end-effectors for robotic harvesting of sweet-pepper. Acta Hortic. 2016, 1130, 567-574. [CrossRef]

20. Dimeas, F.; Sako, D.V.; Moulianitis, V.C.; Aspragathos, N.A. Design and fuzzy control of a robotic gripper for efficient strawberry harvesting. Robotica 2015, 33, 1085-1098. [CrossRef]

21. Yaguchi, H.; Nagahama, K.; Hasegawa, T.; Inaba, M. Development of an autonomous tomato harvesting robot with rotational plucking gripper. In Proceedings of the 2016 IEEE/RSJ International Conference on Intelligent Robots and Systems (IROS), Daejeon, Korea, 9-14 October 2016; Volume 9, pp. 652-657. [CrossRef]

22. Glick, P.; Suresh, S.A.; Ruffatto, D.; Cutkosky, M.; Tolley, M.T.; Parness, A. A Soft Robotic Gripper with Gecko-Inspired Adhesive. IEEE Robot. Autom. Lett. 2018, 3, 903-910. [CrossRef]

23. Takahashi, T.; Kikuchi, S.; Suzuki, M.; Aoyagi, S. Octopus-bioinspired Vacuum Gripper with Micro Bumps. J. Robot. Soc. Jpn. 2017, 35, 62-69. [CrossRef]

24. Tomokazu, T.; Kikuchi, S.; Suzuki, M.; Aoyagi, S. Vacuum gripper imitated octopus sucker-effect of liquid membrane for absorption. In Proceedings of the 2015 IEEE/RSJ International Conference on Intelligent Robots and Systems (IROS), Hamburg, Germany, 28 September-3 October 2015; pp. 2929-2936.

25. Brown, E.; Rodenberg, N.; Amend, J.; Mozeika, A.; Steltz, E.; Zakin, M.R.; Lipson, H.; Jaeger, H.M. Universal robotic gripper based on the jamming of granular material. Proc. Natl. Acad. Sci. USA 2010, 107, 18809-18814. [CrossRef]

26. Shintake, J.; Cacucciolo, V.; Floreano, D.; Shea, H. Soft Robotic Grippers. Adv. Mater. 2018, 30, e1707035. [CrossRef]

27. Abundant Robotics. Apple-Picking Robot Prepares to Competer for Farm Jobs. [EB/OL] 2017, 5, 8. Available online: https://www.sohu.com/a/139050700_355045 (accessed on 23 August 2021).

28. Ma, S.C.; Xie, R.P.; Huang, Q.; Liu, M. Experimental study on the interaction force between ground-wheel and soil of dibble-type transplanter. J. Agric. Mech. Res. 2018, 40, 158-161, 166.

29. Hao, Y.; Gong, Z.; Xie, Z.; Guan, S.; Yang, X.; Wang, T.; Wen, L. A Soft Bionic Gripper with Variable Effective Length. J. Bionic Eng. 2018, 15, 220-235. [CrossRef]

30. Hao, Y.; Wang, T.; Ren, Z.; Gong, Z.; Wang, H.; Yang, X.; Guan, S.; Wen, L. Modeling and experiments of a soft robotic gripper in amphibious environments. Int. J. Adv. Robot. Syst. 2017, 14, 14. [CrossRef]

31. Mazzolai, B.; Laschi, C.; Cianchetti, M.; Patane, F.; Bassi-Luciani, L.; Izzo, I.; Dario, P. Biorobotic Investigation on the Muscle Structure of an Octopus Tentacle. In Proceedings of the 2007 29th Annual International Conference of the IEEE Engineering in Medicine and Biology Society, Montréal, QC, Canada, 20-24 July 2007; Volume 2007, pp. 1471-1474.

32. Tramacere, F.; Beccai, L.; Sinibaldi, E.; Laschi, C.; Mazzolai, B. Adhesion Mechanisms Inspired by Octopus Suckers. Procedia Comput. Sci. 2011, 7, 192-193. [CrossRef]

33. Yekutieli, Y.; Sagiv-Zohar, R.; Aharonov, R.; Engel, Y.; Hochner, B.; Flash, T. Dynamic Model of the Octopus Arm. I. Biomechanics of the Octopus Reaching Movement. J. Neurophysiol. 2005, 94, 1443-1458. [CrossRef] [PubMed]

34. Gutfreund, Y.; Flash, T.; Fiorito, G.; Hochner, B. Patterns of Arm Muscle Activation Involved in Octopus Reaching Movements. J. Neurosci. 1998, 18, 5976-5987. [CrossRef]

35. Kier, W.M.; Smith, K.K. Tongues, tentacles and trunks: The biomechanics of movement in muscular-hydrostats. Zool. J. Linn. Soc. 1985, 83, 307-324. [CrossRef]

36. Yekutieli, Y.; Sagiv-Zohar, R.; Hochner, B.; Flash, T. Dynamic Model of the Octopus Arm. II. Control of Reaching Movements. J. Neurophysiol. 2005, 94, 1459-1468. [CrossRef] [PubMed]

37. Navas, E.; Fernández, R.; Sepúlveda, D.; Armada, M.; Gonzalez-De-Santos, P. Soft Grippers for Automatic Crop Harvesting: A Review. Sensors 2021, 21, 2689. [CrossRef] [PubMed]

38. Chang, L.; Na, L.; Ja, L. Dynamic simulation analysis of ADAMS on pneumatic flexible strawberry picking manipulator. Chin. Sci. Pap. 2017, 12, 390-393.

39. Marchese, A.D.; Katzschmann, R.K.; Rus, D. A recipe for soft fluidic elastomer robots. Soft Robot. 2015, 2, 7-25. [CrossRef] [PubMed] 